\title{
A Spoonful of Mind-Body Medicine: If a Little Is Good, Is More Better?
}

\begin{abstract}
Editor's Note: Which of us who live time pressed have not wanted to take it out on the time we seek to reserve for mindfulness practice-by reducing it? Or wished and hoped that somehow mindfulness benefits might come from, paradoxically, engaging in less of a good thing? In this column in the continuing series from the Osher Center for Integrative Medicine at Harvard Medical School and the Brigham and Women's Hospital, the trio of researchers turn their attention to the topic of dosing. They explore dose-related changes in interleukin (IL)-6 in alcohol use disorder, the value of an app's individualized mini doses, and time investment in t'ai chi practice. Mindfulness slackers may not like the findings. - John Weeks, Editor-in-Chief, JACM
\end{abstract}

\section{Is Mindfulness Training for Alcohol Dependence Dose Dependent?}

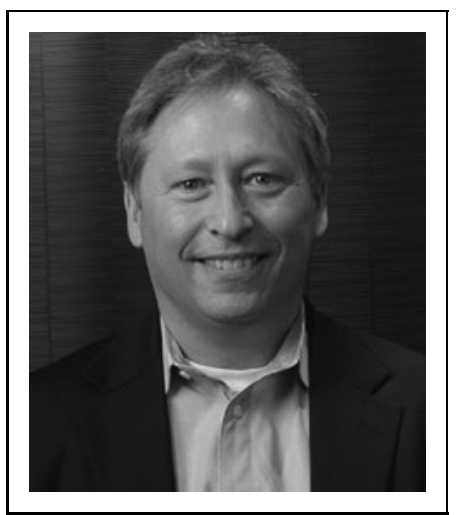

Peter M. Wayne, PhD

Interim Director and Director of Research

Osher Center for Integrative Medicine

Harvard Medical School and Brigham and Women's Hospital
$\mathbf{U}$ NLIKE TYPICAL PHARMACOLOGIC interventions that undergo formal dosing studies prior to being tested for efficacy in clinical trials, nonpharmacologic interventions such as mindfulness programs are rarely evaluated in a doseresponse fashion. In addition to informing the optimal delivery of a specific program for a given target population, when paired with objective biomarkers, information on dose response can inform the validity of an intervention and provide insight into mechanisms of action.

A growing body of research suggests that mindfulnessbased techniques show promise for treating alcohol use disorder (AUD). Research also supports that the negative health effects of chronic heavy alcohol take place, in part, via modulation of the body's immune system and inflammatory physiology. In particular, alcohol-induced elevated levels of the pro-inflammatory cytokine IL-6 have been implicated in an increased risk for hepatic disease, as well as coronary artery disease, some cancers, and premature death. However, evidence linking mindfulness training with effects on IL-6 has produced mixed results. One explanation for this heterogeneity is that group averages, typically based on smaller studies, may have obscured meaningful variation among participants, some of whom are more adherent to protocols than others. 
In this study, researchers based at the University of Wisconsin, Madison, employed secondary analysis to test the hypothesis that between-person variability in engagement with mindfulness training (i.e., formal mindfulness practice time) is associated with between-person variability in changes in serum IL-6. ${ }^{1}$ The parent trial was a 2 -arm, waitlist controlled study $(n=123)$ evaluating a validated Mindfulness-Based Relapse Prevention for Alcohol Dependence (MBRP-A) program on alcohol consumption. ${ }^{2}$ The secondary analysis was limited to 72 alcohol-dependent adults $\left(M_{\text {age }}=43.4\right.$ years, $63.9 \%$ male, $93.1 \%$ white) who received a minimum dose (i.e., at least four sessions) of MBRP-A either at the start of the trial $(n=46)$ or after a 26-week delay (i.e., in the waitlist; $n=26$ ). IL-6 concentrations were assessed from serum blood samples.

Findings suggest that IL-6 concentrations did not significantly change from pre to post intervention for the full sample, paralleling the null clinical finding in the parent trial. However, greater mindfulness practice time was significantly associated with reduced IL-6 levels $(r=-0.27)$, a small to moderate effect following Cohen's classification. This association was robust to multiple sensitivity analyses, including those accounting for intervention timing (i.e., immediate or after the 26-week delay), demographic characteristics, and changes in mindful awareness, obsessive-compulsive drinking, and depressive symptoms. However, of note, the association between practice time and IL- 6 changes was not significant when omitting the minimum treatment dose requirement (i.e., attending 4 sessions), suggesting a baseline minimal threshold dose of required training.
Clear limitations of this study include its small sample size and limited statistical power, biases associated with the use of participants from both the initial exposure and delayed waitlist group (despite accounting for this in models), and, most importantly, the post hoc nature of this exploratory study. Nevertheless, the results suggest that the level of engagement in mindfulness training may predict changes in inflammatory pathophysiology in adults with alcohol dependence, adding evidence to the value of mindfulnessbased programs for AUD, and suggesting biological processes to target (i.e., inflammation physiology) to inform mechanistic models. Future studies employing designs that experimentally control for varying doses/exposures and collect more information on patient engagement beyond practice time, that is, levels of physiological and psychological immersion during practice (quality in addition to quantity), may further inform the clinical and immune system benefits of mind-body practices for AUD and substance use problems in general.

Citations: 1. McClintock AS, Goldberg SB, Coe CL, et al. Mindfulness practice predicts interleukin- 6 responses to a mindfulness-based alcohol relapse prevention intervention. J Subst Abuse Treat 2019;105:57-63.

2. Zgierska AE, Burzinski CA, Mundt MP, et al. Mindfulness-based relapse prevention for alcohol dependence: findings from a randomized controlled trial. J Subst Abuse Treat 2019;100:8-17.

\title{
Dial Up, Dial Down: Individualized Adjusted Mini Doses of Mobile Meditation
}

\author{
Gloria Y. Yeh, MD, MPH \\ Director, Research Fellowship in Integrative Medicine \\ Director of Mind-Body Research \\ Division of General Medicine and Primary Care \\ Beth Israel Deaconess Medical Center \\ Harvard Medical Center
}

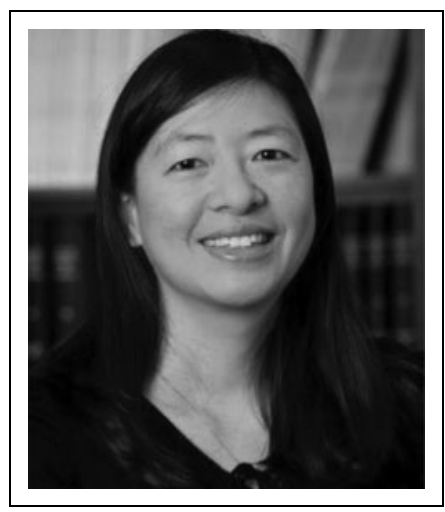

D GITAL MEDITATION MOBILE APPS and other web-based online delivery of mind-body programs are increasingly popular and available. Overall, studies have been promising for increasing reach while maintaining benefit. However, just as with in-person training programs, questions regarding appropriate and best dose remain. How does quality and duration of practice impact outcomes? What is optimal dose of meditation, and how do we manipulate it and study dose optimization in the digital environment?

A recent study published in Nature Human Behavior describes a mobile software platform that takes an inter- esting spin on manipulation of practice dose. Unlike most meditation apps that simply offer set guided meditations through mobile delivery, this new digital platform integrates principles of neuroplasticity and closed-loop feedback to enhance cognition. The app, called MediTrain, begins like others with a lesson and instructions on mindfulness (e.g., to focus attention on the breath). It then distinguishes itself by including a "training trial" followed by the subject's self-introspection and self-report of mind wandering during that trial. The closed-loop aspect of the app uses an adaptive staircase algorithm to adjust the difficulty of the 
next trial, increasing or decreasing the duration depending on the success of the last trial.

In this randomized, double-blind, placebo-controlled study, Ziegler et al. ${ }^{1}$ randomized 59 healthy young adults to receive at-home training with the MediTrain program or an expectancy-matched placebo program consisting of three commercial non-meditation apps (foreign language learning, animated t'ai chi steps, and logic games). While participants were asked to spend about $10 \mathrm{~min}$ per day on the program app(s) using a provided iPad (5 days a week for 6 weeks), the closed-loop program provided varying individualized mini doses of practice time.

Overall, with this approach, participants in the treatment group gradually increased their "successful" practice times each day (average from $20 \mathrm{~s}$ on day 1 to 6 min after 25 days). There were reported gains in sustained attention (decrease in variability in response time, $R T_{\mathrm{var}}$, across trials on a vigilance task $\Delta=-7.98 \mathrm{~ms}, p=0.002$ vs. no change in placebo $\Delta=1.1 \mathrm{~ms}, p=0.78$ ), as well as gains in working memory (between-group improvement in ability to maintain an accurate mental representation of items in working memory in the presence or absence of distracting stimuli; Cohen's $d=0.66$, $p=0.04)$.

Participants underwent electroencephalography recordings during the vigilance task to examine neural mechanisms of sustained attention. Interestingly, improvements were associated with positive changes in key neural signatures of attentional control (frontal theta inter-trial coherence and parietal P3b latency). The investigators also reported a significant relationship between improvement of practice times and sustained attention (Pearson $\mathrm{r}_{18}=-0.59, p=0.01$ ), such that participants who advanced the most in the amount of focused practice time showed the greatest decrease in $R T_{\text {var }}$. Taking advantage of technology, this investigative group was able to adopt an individualized approach to dose optimization.

Perhaps the best dose is one that is self-paced and internally directed rather than externally imposed. Intuitively and pragmatically, this may be what happens every day in the real world. We expect that participants who can achieve quality practice time are more likely to reap benefits and likely in a dose-response fashion. Yet, the path toward progress and sustained practice might be a gradual iterative trajectory based on an individual's prior experience. The development of further technology that bridges the science of meditation with these concepts of dose in innovative, adaptive, and measurable ways may not only facilitate better clinical optimization and uptake of mind-body training but also allow a platform for important mechanistic dose research to move the field forward.

Citation: 1. Ziegler DA, Simon AJ, Gallen CL, et al. Closed-loop digital meditation improves sustained attention in young adults. Nat Hum Behav 2019;3:746-757.

\section{How Much T'ai Chi Do I Really Need? Learning to Prescribe Mind-Body Medicine}

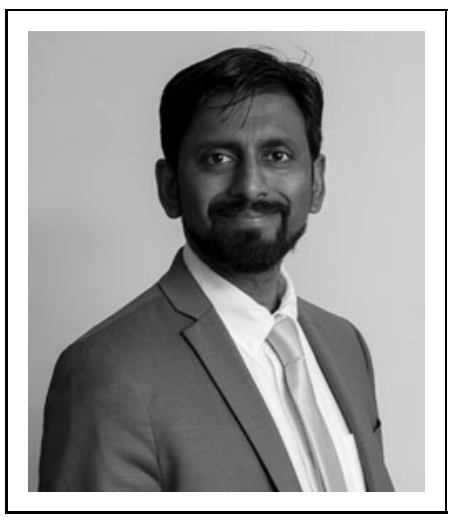

\author{
Darshan H. Mehta, MD, MPH \\ Medical Director, Benson-Henry Institute for Mind Body Medicine \\ Massachusetts General Hospital \\ Director of Education \\ Osher Center for Integrative Medicine \\ Harvard Medical School and Brigham and Women's Hospital
}

$\mathbf{U}$ NTIL NOW, MOST RESEARCH studies have focused on whether mind-body practice has a clinical effect. While it is important to accumulate such data, in practice, most patients want to know how much practice is needed to have the desired effect. And health-care providers want to be able to prescribe more precisely. They want to know the right dose needed to have the desired clinical effect. The sages of different wisdom traditions will ubiquitously say that this means a lifetime of practice. However, for what providers and patients are seeking, this is a nebulous and perhaps frustrating response. The best we can do is infer from the study itself. Even when one aggregates all mind-body practices that have been studied, those that examine dose and frequency are increasingly relevant.
In this secondary analysis of a large-scale single-blinded randomized trial by Lee et al., ${ }^{1}$ funded by the National Institutes of Health, 182 subjects with osteoarthritis were randomized to either 12 weeks of t'ai chi or physical therapy (PT). Each 1-h t'ai chi class met twice per week, delivered as a standardized protocol by experienced t'ai chi instructors. Following national guidelines, the PT intervention was structured as 6 weeks of one-to-one treatment (30 min twice per week) followed by 6 weeks of monitored home treatment ( $30 \mathrm{~min} 4$ times per week). The authors were specific in making sure that both treatment arms were equivalent in treatment duration.

The study investigators used traditional criteria set by the American College of Rheumatology for knee osteoarthritis. 
In addition, they were very specific in having individuals in the study who had no experience with PT or t'ai chi (or any other complementary medicine program) in the past year. The primary outcome measures were the weekly collection of Western Ontario and McMaster Osteoarthritis Index (WOMAC) pain and function scores. This is a widely used and validated self-report measure in studies on the treatment of knee osteoarthritis, including pharmacologic treatments. Two clinically meaningful response thresholds were used: (1) $>20 \%$ improvement in pain and (2) $>50 \%$ improvement in pain and function. These thresholds were established by outside guidelines. Attendance was counted if a study subject participated in at least one session that week. The participants were largely female, white, and educated. The two groups were very evenly matched in baseline characteristics.

In their response, both groups demonstrated a similar linear relationship regarding improvements in pain and function. In the t'ai chi group, one attendance week resulted in a 11.4-point improvement in WOMAC pain and a 40.6-point improvement in function. In the PT group, one attendance week resulted in a 9.3-point improvement in WOMAC pain and a 32.4-point improvement in function. As per the parent study, these improvements were similar and clinicallymeaningful. The time to respond for a $20 \%$ improvement in pain was 2 attendance weeks for t'ai chi and PT. The time to respond for a $50 \%$ improvement in pain or function was 4 weeks of PT or 5 weeks of t'ai chi. This difference was not found to be significantly different. Finally, in a multivariate analysis, the study authors found that outcome expectations were independently associated with function response at any given point in time of the 12 -week interventions.

This study is clever for several reasons. It was designed as a comparative effectiveness study with clinically meaningful outcomes in a highly prevalent condition. Osteoarthritis is a common disability that especially afflicts the elderly. It is a leading cause of disability worldwide. Second, the study showed that the more you participate, the more you benefit. Finally, expectation matters independent of exercise adherence. Expectation was found to predict the treatment outcome at any given attendance week. An additional intriguing characteristic is that if this study were guised as a pharmacologic intervention, it would satisfy the requirements for the Food and Drug Administration's drug approval process.

And so, what does this mean? Obviously, future studies are needed to replicate these findings. And it will be interesting to see if these effects are seen in other movementbased mind-body practices such as yoga. In addition, it will be interesting to understand overall costs and health-care utilization patterns, especially in a condition that causes so much disability. However, from a practical perspective, this study can already begin to inform clinical practice that can be customized to the patient. For patients who are more suited to one-to-one care, physical therapy might be the appropriate option. However, for patients who are socially isolated, learning t'ai chi in a group-based setting may be more prudent. And so, here is my exercise prescription for people with osteoarthritis (and its associated rationale):

(1) Attend at least two 1-h sessions of group t'ai chi or individual PT (because as little as 2 attendance weeks of treatment can provide benefit to patients).

(2) Try to attend five 1-h sessions of group t'ai chi or individual PT (because this reduces pain and improve function by half).

(3) Encourage and guide your patient (because this can affect the outcome).

Citation: 1. Lee AC, Harvey WF, Price LL, et al. Doseresponse effects of t'ai chi and physical therapy exercise interventions in symptomatic knee osteoarthritis. $P M R$ 2018;10:712-723.

Address correspondence to: Peter M. Wayne, PhD Osher Center for Integrative Medicine Brigham and Women's Hospital and Harvard Medical School 900 Commonwealth Avenue, 3rd Floor Boston, MA 02215

E-mail: pwayne@partners.org 